\title{
Designs and Full-Rank STBCs from DFT Domain Description of Cyclic Codes
}

\author{
U. Sripati, V. Shashidhar and B. Sundar Rajan \\ Dept. of Electrical Communication Engineering \\ Indian Institute of Science, Bangalore 560012, INDIA \\ \{sripati@protocol., shashidhar@protocol., bsrajan@\}ece.iisc.ernet.in
}

\begin{abstract}
Viewing an $n$ length vector over $F_{q^{m}}$ as an $m \times n$ matrix over $F_{q}$, by expanding each entry of the vector with respect to a basis of $F_{q^{m}}$ over $F_{q}$, the rank weight of the $n$ length vector over $F_{q^{m}}$ is the rank of the corresponding $m \times n$ matrix over $F_{q}$. It is known that under some conditions, $n$-length cyclic codes over $F_{q^{m}},\left(n \mid q^{m}-1\right.$ and $\left.m \leq n\right)$ have full rank. In this paper, using this result we obtain a design using which we construct full-rank Space-Time Block Codes (STBCs) for $m$ transmit antennas over signal sets matched to $F_{q}$ where $q=2$ or $q$ is a prime of the form $4 k+1$. We also propose a construction of STBCs using $n$-length cyclic codes over $F_{q^{m}}$, for $r$ transmit antennas, where $r \leq n$ and $r \mid m$.
\end{abstract}

\section{Extended Summary}

The characterization of cyclic codes using the Rank metric has been studied in [1]. In this paper, we use the main result of [1] to obtain full-rank STBCs.

Definition 1 A rate- $k / n, n \times l$ linear design over a field $F$ is an $n \times l$ matrix with all its entries $F$-linear combinations of $k$ variables which are allowed to take values from the field $F$.

Let $(n, q)=1$ and $n \mid q^{m}-1$, where $q$ is either 2 or a prime of the form $4 k+1$. Let $[j]_{n}$ be a $q$-cyclotomic coset of $I_{n}$ of size $m$. By restricting $A_{j}, j \in[j]_{n}$, to $F_{q^{m}}$ and constraining all other transform components to zero, we have a $n$-length code over $F_{q^{m}}$ whose codewords are of the form, $\left[\begin{array}{llllll}A_{j} & \alpha^{-j} A_{j} & \alpha^{-2 j} A_{j} & \cdots & \alpha^{-(n-1) j} A_{j}\end{array}\right]$ where $\alpha$ is a primitive $n$-th root of unity in $F_{q^{m}}$ and $A_{j} \in F_{q^{m}}$. Viewing $A_{j}$ as a $m$-length column vector over $F_{q}$, the codewords can be viewed as $m \times n$ matrices over $F_{q}$ given by

$$
\left[\begin{array}{ccccc}
A_{0,0} & A_{0,1} & A_{0,2} & \cdots & A_{0, n-1} \\
A_{1,0} & A_{1,1} & A_{1,2} & \cdots & A_{1, n-1} \\
\vdots & \vdots & \vdots & \vdots & \vdots \\
A_{m-1,0} & A_{m-1,1} & A_{m-1,2} & \cdots & A_{m-1, n-1}
\end{array}\right]
$$

where $\alpha^{-k j} A_{j}=\sum_{i=0}^{m-1} A_{i, k} \beta^{i}, A_{i, k} \in F_{q}$ and $\beta$ is a primitive element of $F_{q^{m}}$. Notice that (1) is a design over $F_{q}$ and the variables are allowed to take values from a signal set matched to $F_{q}$. Also, note that this is possible for any linear code, however only for cyclic codes we have information about the rank. To obtain an STBC from the above design, we have to map the elements of $F_{q}$ into the complex field such that the rank is preserved. The following maps have been proposed for the same:

Case 1. $q=2$, Hammons et al. [2]: A rank preserving map given by Hammons et al.[2] is as follows : $0 \mapsto+1,1 \mapsto-1$.

\footnotetext{
${ }^{1}$ This work was partly funded by the DRDO-IISc program on Advanced Research in Mathematical Engineering through a grant to B.S.Rajan.
}

Thus, by using the above map, we obtain STBCs over BPSK signal sets.

Case 2. $q=4 k+1$, Lusina et al. [3]: Let $q$ be a prime of the form $q=4 k+1$. Then, it is known that $q=u^{2}+v^{2}$ for some integers $u$ and $v$. Let $\Pi^{\prime}=u-i v$. Then $w$ modulo $\Pi$ of any integer $w$ is defined as,

$\zeta=w \operatorname{modulo} \Pi=w-\left[\frac{w \Pi^{\prime}}{\Pi \Pi^{\prime}}\right] \Pi$ where [.] performs the operation of rounding to the nearest Gaussian number. In [3], it is proved that the Gaussian numbers modulo $\Pi$ form a field, $G_{\Pi}=\left\{\zeta_{0}=0, \zeta_{1}=1, \zeta_{2}, \cdots, \zeta_{q-1}\right\}$ where $\zeta_{i}=i \bmod \Pi$.

Example 1 Let the number of transmit antennas be 2 and $q=5$. Then, we take $n=3$ and $m=2$. The 5 -cyclotomic coset of 1 is $\{1,2\}$. Thus, allowing only $A_{1}$ to take values from $F_{25}$ and constraining other components to zero, we have a full-rank $2 \times 3$ STBC with codewords of the form $\left[\begin{array}{lll}\xi\left(a_{0}\right) & \xi\left(3 a_{0}+a_{1}\right) & \xi\left(a_{0}+4 a_{1}\right) \\ \xi\left(a_{1}\right) & \xi\left(a_{0}+2 a_{1}\right) & \xi\left(3 a_{0}+3 a_{1}\right)\end{array}\right]$ where $a_{0}, a_{1} \in F_{5}$ and $\xi: F_{5} \mapsto G_{1+2 i}$.

STBCs for $r$ transmit antennas, $r \mid m$ : Let $q$ be a prime of the form $4 k+1$. Then, we have the map $\xi: F_{q} \mapsto G_{\pi}$. Now, let $F_{q^{r}}=F_{q}[\beta]$, where $\beta$ is a root of a polynomial $f$ irreducible over $F_{q}$ and of degree $r$.

Theorem 1 Consider the map $\xi^{\prime}: F_{q^{r}} \mapsto G_{\pi}[\gamma]$ given by $\left.\xi^{\prime}\right|_{F_{q}}=\xi$ and $\xi^{\prime}(\beta)=\gamma$, where $\gamma$ is a root of the polynomial $\xi(f)$ in the complex field. Then, the map $\xi^{\prime}$ is a ring isomorphism. And hence $V \in F_{q^{r}}^{n \times n}$ is non-singular in $F_{q^{r}}$ if and only if $\xi^{\prime}(V)$ is non-singular in the complex field.

The usage of the above theorem to construct full-rank STBCs is illustrated in the following example.

Example 2 Let $n=5$ and $q=17$. Then, we have $m=4$. The 17-cyclotomic coset of 1 is $\{1,2,3,4\}$. So, allowing $A_{1}$ to take values from $F_{17^{4}}$ and constraining all other components to zero, we get a length 5 cyclic code $\mathbf{C}$ over $F_{17^{4}}$. Expand each component into a 2-length column vector over $F_{17^{2}}$. The $F_{17^{2}}$-rank of this code is equal to 2 because, the size of the $17^{2}$-cyclotomic coset $\{1,4\}$ of 1 is 2 . The entries of the codewords over $F_{17^{2}}$ will be of the form $a_{0}+a_{1} \beta$, where $\beta$ is a primitive element of $F_{17^{2}}$ and a root of an irreducible polynomial polynomial $f \in F_{17}[x]$ of degree 2 . Let $\gamma$ be a root of the polynomial $\xi(f)$ in the complex field. Then, using the map $\xi^{\prime}$, we obtain a full-rank $S T B C$ for 2 transmit antennas.

\section{REFERENCES}

[1] U.Sripati and B.Sundar Rajan, " On the Rank Distance of Cyclic Codes," Proc. IEEE International Symposium on Information Theory, Yokohoma, Japan, June-July 2003, p.72.

[2] A.Roger Hammons Jr. and Hesham El Gamal, "On the Theory of Space Time Codes for PSK modulation," IEEE Trans. on Information Theory, vol.46, No.2, March-2000, 524-542.

[3] Paul Lusina, E.Gabidulin and Martin Bossert, "Maximum Rank Distance Codes as Space Time Codes," IEEE Trans. on Information Theory, vol.49, No.10, Oct. 2003, pp. 2757-2760. 